This paper is published as part of a $P C C P$ themed issue on characterisation of adsorbed species

\section{Guest Editors: Petr Nachtigall and Carlos Otero Arean}

Editorial

Themed Issue on characterisation of adsorbed species

Petr Nachtigall and Carlos Otero Arean, Phys. Chem. Chem. Phys., 2010

DOI: $10.1039 / \mathrm{c0cp90026e}$

Perspective

Hydroxyapatite as a key biomaterial: quantummechanical simulation of its surfaces in interaction with biomolecules

Marta Corno, Albert Rimola, Vera Bolis and Piero Ugliengo, Phys. Chem. Chem. Phys., 2010

DOI: $\underline{10.1039 / \mathrm{c} 002146 f}$

Communications

Hydrogen induced $\mathrm{CO}$ activation on open $\mathrm{Ru}$ and $\mathrm{Co}$ surfaces

Sharan Shetty and Rutger A. van Santen, Phys. Chem.

Chem. Phys., 2010

DOI: $10.1039 / \mathrm{b} 926731 \mathrm{j}$

Large heterogeneity of Brønsted acid sites in ASA and

USY zeolites: evidencing a third acidic component

Olivier Cairon, Phys. Chem. Chem. Phys., 2010

DOI: 10.1039/c000991a

Papers

Simultaneous adsorption of benzene and dioxygen in CuHY zeolites as a precursor process to the aerobic oxidation of benzene to phenol

Shampa Santra, Hermann Stoll and Guntram Rauhut, Phys. Chem. Chem. Phys., 2010

DOI: $10.1039 / \mathrm{b} 921531 \mathrm{j}$

Physisorption of aromatic organic contaminants at the surface of hydrophobic/hydrophilic silica geosorbents a B3LYP-D modeling study

Albert Rimola, Bartolomeo Civalleri and Piero Ugliengo,

Phys. Chem. Chem. Phys., 2010

DOI: $10.1039 / \mathrm{c} 000009 \mathrm{~d}$

Formation of $\mathrm{O}$ adatom pairs and charge transfer upon $\underline{\mathrm{O}}_{2}$ dissociation on reduced $\mathrm{TiO}_{2}(110)$

Yingge Du, Nathaniel A. Deskins, Zhenrong Zhang, Zdenek

Dohnalek, Michel Dupuis and Igor Lyubinetsky, Phys.

Chem. Chem. Phys., 2010

DOI: $10.1039 / \mathrm{c} 000250 \mathrm{j}$

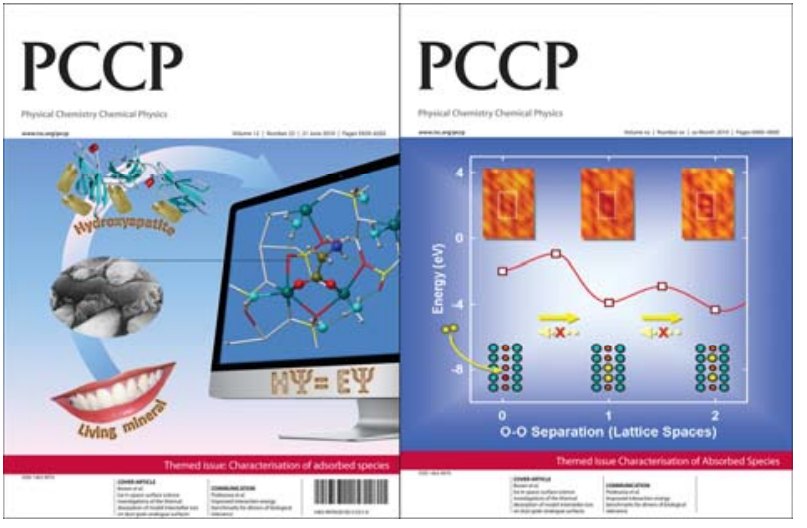

Study of polycyclic aromatic hydrocarbons adsorbed on graphene using density functional theory with empirical dispersion correction

Olga V. Ershova, Timothy C. Lillestolen and Elena

Bichoutskaia, Phys. Chem. Chem. Phys., 2010

DOI: $\underline{10.1039 / \mathrm{c000370k}}$

DFT/CC investigation of physical adsorption on a graphite (0001) surface

Miroslav Rube š, Jiří Kysilka, Petr Nachtigall and Ota Bludský, Phys. Chem. Chem. Phys., 2010

DOI: $10.1039 / \mathrm{c} 001155$ j

Monitoring the interaction of adsorbates on metal surfaces by surface site engineering: the case of ethoxy on $\mathrm{Cu}, \mathrm{Pd}, \mathrm{Ag}$ and $\mathrm{Au}$ reqular and stepped surfaces

Juan Radilla, Mercé Boronat, Avelino Corma and Francesc Illas, Phys. Chem. Chem. Phys., 2010

DOI: $10.1039 / \mathrm{c000405g}$

Adsorption of oxygen on copper in Cu/HZSM5 zeolites Augusta Bianca Ene, Matthias Bauer, Tanja Archipov and Emil Roduner, Phys. Chem. Chem. Phys., 2010

DOI: $10.1039 / \mathrm{c} 000750 \mathrm{a}$

Elucidation of consistent enantioselectivity for a homologous series of chiral compounds in homochiral metal-organic frameworks

Xiaoying Bao, Linda J. Broadbelt and Randall Q. Snurr, Phys. Chem. Chem. Phys., 2010

DOI: $\underline{10.1039 / \mathrm{c000809e}}$

A DFT study of PtAu bimetallic clusters adsorbed on MgO/Ag(100) ultrathin films

Sabrina Sicolo and Gianfranco Pacchioni, Phys. Chem. Chem. Phys., 2010

DOI: $10.1039 / \mathrm{c000841a}$

CO as an IR probe molecule for characterization of copper ions in a basolite C300 MOF sample

Nikola Drenchev, Elena Ivanova, Mihail Mihaylov and Konstantin Hadjiivanov, Phys. Chem. Chem. Phys., 2010 DOI: $10.1039 / \mathrm{c} 000949 \mathrm{k}$

Existence of dual species composed of $\mathrm{Cu}^{+}$in $\mathrm{CuMFI}$ being bridged by $\mathrm{C}_{2} \mathrm{H}_{2}$

Atsushi Itadani, Takashi Yumura, Takahiro Ohkubo, Hisayoshi Kobayashi and Yasushige Kuroda, Phys. Chem. Chem. Phys., 2010

DOI: $10.1039 / \mathrm{c000967a}$

Benchmark calculations of water-acene interaction energies: Extrapolation to the water-graphene limit and assessment of dispersion-corrected DFT methods Glen R. Jenness, Ozan Karalti and Kenneth D. Jordan, 
Phys. Chem. Chem. Phys., 2010

DOI: $10.1039 / \mathrm{c} 000988 \mathrm{a}$

Adsorption of light hydrocarbons in the flexible MIL53(Cr) and rigid MIL-47(V) metal-organic frameworks: a combination of molecular simulations and microcalorimetry/gravimetry measurements

N. Rosenbach Jr, A. Ghoufi, I. Déroche, P. L. Llewellyn, T. Devic, S. Bourrelly, C. Serre, G. Férey and G. Maurin, Phys. Chem. Chem. Phys., 2010

DOI: $10.1039 / \mathrm{c} 001173 \mathrm{~h}$

Role of dispersive interactions in the $\mathrm{CO}$ adsorption on MgO(001): periodic B3LYP calculations augmented with an empirical dispersion term

Bartolomeo Civalleri, Lorenzo Maschio, Piero Ugliengo and Claudio M. Zicovich-Wilson, Phys. Chem. Chem. Phys., 2010

DOI: $\underline{10.1039 / \mathrm{c001192d}}$

Nature and role of surface carbonates and bicarbonates in CO oxidation over $\mathrm{RuO}_{2}$

Hangyao Wang and William F. Schneider, Phys. Chem.

Chem. Phys., 2010

DOI: $10.1039 / \mathrm{c001683g}$

Experimental and theoretical determination of adsorption heats of $\mathrm{CO}_{2}$ over alkali metal exchanged ferrierites with different SilAl ratio

Arnost Zukal, Angeles Pulido, Barbara Gil, Petr Nachtigall, Ota Bludský, Miroslav Rubeš and Jił̌́ Cejka, Phys. Chem.

Chem. Phys., 2010

DOI: 10.1039/c001950j

Six-dimensional dynamics study of reactive and non reactive scattering of $\mathrm{H}_{2}$ from $\mathrm{Cu}(111)$ using a chemically accurate potential energy surface

C. Díaz, R. A. Olsen, D. J. Auerbach and G. J. Kroes, Phys. Chem. Chem. Phys., 2010

DOI: $10.1039 / \mathrm{c} 001956 \mathrm{a}$

Azobenzene versus $3,3^{\prime}, 5,5^{\prime}$-tetra-tert-butyl-azobenzene (TBA) at Au(111): characterizing the role of spacer groups

Erik R. McNellis, Christopher Bronner, Jörg Meyer, Martin Weinelt, Petra Tegeder and Karsten Reuter, Phys. Chem. Chem. Phys., 2010

DOI: $10.1039 / \mathrm{c001978j}$

FTIR spectroscopy and thermodynamics of $\mathrm{CO}$ and $\mathrm{H}_{2}$ adsorbed on $\gamma-, \delta$ - and $\alpha-\mathrm{Al}_{2} \mathrm{O}_{3}$

Evgeniy N. Gribov, Olena Zavorotynska, Giovanni Agostini, Jenny G. Vitillo, Gabriele Ricchiardi, Giuseppe Spoto and Adriano Zecchina, Phys. Chem. Chem. Phys., 2010

DOI: $10.1039 / \mathrm{c} 002031 \mathrm{c}$

A highly ordered, aromatic bidentate self-assembled monolayer on Au(111): a combined experimental and theoretical study

Xia Stammer, Katrin Tonigold, Asif Bashir, Daniel Käfer, Osama Shekhah, Christian Hülsbusch, Martin Kind, Axel Groß and Christof Wöll, Phys. Chem. Chem. Phys., 2010 DOI: $10.1039 / \mathrm{c002215m}$

Modelling active sites for the Beckmann rearrangement reaction in boron-containing zeolites and their interaction with probe molecules

Inés Lezcano-González, Alejandro Vidal-Moya, Mercedes Boronat, Teresa Blasco and Avelino Corma, Phys. Chem.

Chem. Phys., 2010

DOI: $10.1039 / \mathrm{c} 002427 \mathrm{a}$
A FTIR search for linkage isomerism of $\mathrm{CN}$ ions adsorbed on oxides and zeolites

A. A. Tsyganenko, A. M. Chizhik and A. I. Chizhik, Phys. Chem. Chem. Phys., 2010

DOI: $10.1039 / \mathrm{c} 003942 \mathrm{j}$ 


\title{
Study of polycyclic aromatic hydrocarbons adsorbed on graphene using density functional theory with empirical dispersion correction
}

\author{
Received 11th January 2010, Accepted 15th March 2010 \\ First published as an Advance Article on the web 9th April 2010 \\ DOI: $10.1039 / \mathbf{c 0 0 0 3 7 0 k}$
}

Olga V. Ershova, ${ }^{a}$ Timothy C. Lillestolen $^{b}$ and Elena Bichoutskaia ${ }^{* b}$

The interaction of polycyclic aromatic hydrocarbon molecules with hydrogen-terminated graphene is studied using density functional theory with empirical dispersion correction. The effective potential energy surfaces for the interaction of benzene, $\mathrm{C}_{6} \mathrm{H}_{6}$, naphthalene, $\mathrm{C}_{10} \mathrm{H}_{8}$, coronene, $\mathrm{C}_{24} \mathrm{H}_{12}$, and ovalene, $\mathrm{C}_{32} \mathrm{H}_{14}$, with hydrogen-terminated graphene are calculated as functions of the molecular displacement along the substrate. The potential energy surfaces are also described analytically using the lowest harmonics of the Fourier expansion. It is shown that inclusion of the dispersive interaction, which is the most important contribution to the binding of these weakly bound systems, does not change the shape of the interaction energy surfaces or the value of the barriers to the motion of polycyclic aromatic hydrocarbon molecules on graphene. The potential energy surfaces are used in the estimation of the friction forces acting on the molecules along the direction of motion. These results underpin the modelling, using density functional theory, of electromechanical devices based on the relative vibrations of graphene layers and telescoping carbon nanotubes.

\section{Introduction}

The van der Waals (vdW) dispersive interactions between weakly bound systems are notoriously difficult to compute both in gas phase and in periodic solid structures. They are governed purely by long-range electron correlations, which in small non-covalently bound molecular complexes can be taken into account within the framework of standard $a b$ initio wave function theory, reaching benchmark accuracy in computations based on coupled-cluster methods such as coupled-cluster with single, double and perturbative triple excitations, $\operatorname{CCSD}(\mathrm{T})$. The CCSD(T) methods become computationally expensive for medium-sized systems, and take prohibitively long times for large complexes. The less-expensive second-order Møller-Plesset perturbation theory (MP2) systematically overestimates the binding energies and underestimates intermolecular distances for dispersive $\pi-\pi$ interactions. ${ }^{1,2}$

In condensed matter physics, density functional theory (DFT) is the most widely used approach for calculating electronic structure of medium to large-scale ground-state systems due to its reasonable accuracy and relatively low computational cost. However, a general disadvantage of most commonly used exchange-correlation functionals based on the local (spin) density approximation (LDA) and semi-local generalized gradient approximation (GGA) is that they can not describe long range electron correlations responsible for dispersive interactions. ${ }^{3-5}$ The reason for this failure is that these density functional approximations are derived from the

\footnotetext{
${ }^{a}$ Moscow Institute of Physics and Technology, 141700, Dolgoprudny,

Moscow Region, Russia

${ }^{b}$ Department of Chemistry, University of Nottingham,

University Park, Nottingham, UK NG7 2RD.

E-mail: elena.bichoutskaia@nottingham.ac.uk
}

localized model exchange-correlation holes, whereas the exact exchange-correlation hole is fully nonlocal. Also hybrid functionals, in which DFT is combined with Hartree Fock (HF) theory to incorporate nonlocality of the exchange-correlation hole into the density functional approximations, are unable to describe adequately dispersive interactions.

This can be demonstrated most clearly by calculations of the interlayer cohesive energy in graphite. Graphite is a layered material with highly anisotropic bonding. In-plane, the bonding is dominated by strongly localized covalent $\mathrm{sp}^{2}$ hybridized orbitals, while the interlayer cohesive energy arises from weak nonlocal vdW interactions between the layers. Despite graphite being an abundant material, experiments on the determination of its interlayer cohesion have been scarce. The data are mainly restricted to three experimental values of Benedict et al. ${ }^{6}$ obtained from the study of radial deformations of multi-walled carbon nanotubes, yielding $35_{-10}^{+15} \mathrm{meV} / \mathrm{atom}$; of Girifalco $^{7}$ who reported an exfoliation energy of $43 \mathrm{meV} /$ atom, determined in a heat of wetting experiment; and most recently the result of Zacharia ${ }^{8}$ who obtained an interlayer cohesive energy of graphite of $52 \pm 5 \mathrm{meV} /$ atom in the study of thermal desorption of thin films of polycyclic aromatic hydrocarbon molecules (PAHs) from the surface of graphite.

The computed GGA (PBE) results for graphite give insufficient cohesion energies of as little as $2-5 \mathrm{meV} /$ atom $^{9-12}$ or even predict graphite to be unbound. The LDA method gives much better results for the cohesion energy, ranging from 20 to $35 \mathrm{meV} /$ atom. $^{9-11,13-16}$ The latter values are close to the experimental range, however, the apparent success of the LDA predictions for the interlayer binding in graphite is rather fortuitous. Various authors have proposed to supplement the LDA and GGA DFT calculations of the cohesion energy in graphite with an empirical atom-atom vdW interaction term. 
This term yields an additional long-range attractive potential between layers, incorporating correct long range potentials of the $C_{4} D^{-4}$ and $C_{3} D^{-3}$ forms. ${ }^{9-11,17-19}$ The LDA + vdW and GGA + vdW approaches give consistent results for the interlayer binding energy of graphite in the region of 50 to $65 \mathrm{meV} /$ atom, thus indicating that the weak dispersion forces remain the major contribution to the binding between the layers in graphite.

The investigation of the interlayer interaction in graphite provides a useful starting point not only for the studies of the energetics of other graphitic systems, but also for modelling the relative motion of the components of nested and layered nanomaterials. A number of electromechanical devices have been realised which exploit the remarkable low-friction rotational and sliding capabilities of carbon nanotube walls. ${ }^{20-26}$ Similarly, self-retracting motion of graphite microflakes has been recently observed, in which small flakes of graphite repeatedly move back-and-forth over large islands of highly oriented pyrolytic graphite. ${ }^{27}$ If an accurate quantitative description of the interaction between the walls of carbon nanotubes (or layers of graphitic material) is available, the values for the barriers to their relative motion can be extracted from the interlayer interaction energy surfaces, and subsequently used to compute experimentally measurable quantities such as vibrational frequencies, ${ }^{28}$ threshold and capillary forces, diffusion coefficients and mobilities. ${ }^{29-31}$

In this paper, it is shown that the dispersion interactions are crucial in determination of the binding energies in layered materials, however they do not significantly affect the values of barriers to the relative motion of PAHs on hydrogenterminated graphene. Graphene, a single sheet of graphite, ${ }^{32}$ represents an ideal viewing platform for molecular structures using transmission electron microscopy (TEM) because it provides a robust and low contrast support for molecules and other nanoscale species adsorbed on the surface. Under TEM observation with exposure to an $80 \mathrm{keV}$ electron beam (e-beam) the edges of a graphene sheet continuously change shape, and the high energy of the e-beam transferred to the carbon atoms can cause fragmentation of large sheets of graphene into smaller flake-like graphitic structures. ${ }^{33}$ The flakes adsorbed on a graphene substrate can be visualised in TEM, and their interaction with the underlying graphene substrate can be exploited in nanoscale sensing applications. The study of adsorption and motion of PAHs on hydrogenterminated graphene presented in this paper will elucidate the largely unexplored nature of the interaction of graphene flakes with graphene layer.

\section{Methodology and tests}

In this work, all calculations have been performed using the Q-Chem quantum chemistry package. ${ }^{34}$ For the binding energies, the counterpoise correction ${ }^{35}$ was used to correct for basis set superposition error (BSSE). A long-range corrected (LC) hybrid DFT functional with empirical dispersion correction, $\omega \mathrm{B} 97 \mathrm{X}-\mathrm{D},{ }^{36}$ was used. The $\omega \mathrm{B} 97 \mathrm{X}-\mathrm{D}$ functional is a new generation of the earlier LC hybrid density functional $\omega \mathrm{B} 97 \mathrm{X}^{37}$ which includes an empirical damped atom-atom dispersion correction. The $\omega \mathrm{B} 97 \mathrm{X}$ functional ${ }^{37}$ employs $100 \%$ exact exchange for long-range electron-electron interactions, approximately $16 \%$ short-range exact exchange, a modified B97 exchange density functional for short-range interactions and the B97 correlation density functional ${ }^{38}$ as follows:

$$
E_{\mathrm{xc}}^{\omega \mathrm{B} 97 \mathrm{X}}=E_{\mathrm{x}}^{\mathrm{LR}-\mathrm{HF}}+c_{\mathrm{x}} E_{\mathrm{x}}^{\mathrm{SR}-\mathrm{HF}}+E_{\mathrm{x}}^{\mathrm{SR}-\mathrm{B} 97}+E_{\mathrm{c}}^{\mathrm{B} 97}
$$

where $\omega$ is the parameter which defines the split of the Coulomb operator into the long-range and short-range operators. In the $\omega \mathrm{B} 97 \mathrm{X}-\mathrm{D}$ functional, an empirical atomic pairwise dispersion correction is added to the Kohn-Sham part of the total energy following the general formalism of the DFT-D (density functional theory with empirical dispersion corrections) schemes ${ }^{1,39}$ as:

$$
E^{\mathrm{\omega B} 97 \mathrm{X}-\mathrm{D}}=E_{\mathrm{xc}}^{\mathrm{\omega B} 97 \mathrm{X}}+E_{\mathrm{disp}}
$$

where $E_{\text {disp }}$ is given by

$$
E_{\mathrm{disp}}=-\sum_{i=1}^{N_{\mathrm{at}}-1} \sum_{j=i+1}^{N_{\mathrm{at}}} \frac{C_{6}^{i j}}{R_{i j}^{6}} f_{\mathrm{damp}}\left(R_{i j}\right) .
$$

Here, $N_{\text {at }}$ is the number of atoms in the system, $R_{i j}$ is the interatomic distance between atom $i$ and atom $j$, and $C_{6}^{i j}$ is the dispersion coefficient for a pair of atoms $i$ and $j$, calculated as the geometric mean of the atomic dispersion coefficients $\left(C_{6}^{i j}=\sqrt{C_{6}^{i} C_{6}^{j}}\right) \cdot{ }^{39} \mathrm{~A}$ damping function of the following form:

$$
f_{\text {damp }}\left(R_{i j}\right)=\frac{1}{1+\alpha\left(R_{i j} / R_{r}\right)^{-12}}
$$

is introduced in order to satisfy the condition of zero dispersion correction at short interatomic separations and to provide the correct asymptotic behaviour. This prevents the undesirable divergence of the undamped dispersion correction at small $R_{i j}$ and tends to unity at large $R_{i j}$. The parameter $\alpha$ controls the strength of the dispersion correction, and $R_{r}$ is the sum of van der Waals radii of the atomic pair $i j$, using the atomic van der Waals radii given by Grimme. ${ }^{39}$ All the parameters used in the $\omega \mathrm{B} 97 \mathrm{X}-\mathrm{D}$ functional are re-optimised self-consistently using a least-squares fitting procedure. ${ }^{36}$ The $\omega$ B97X-D functional was tested extensively using various training sets across the applications typical for weakly bound systems, as described in ref. 36. For non-covalent interactions, $\omega$ B97X-D compares favourably to the earlier $\omega \mathrm{B} 97 \mathrm{X}$ and $\mathrm{B} 97$ hybrid functionals, ${ }^{36}$ as well as to other existing DFT-D functionals such as B97-D, B3LYP-D, and BLYP-D. ${ }^{39}$

In ref. 36, the results of the performance tests can be found in the form of tabulated statistical errors. In this paper, the $\omega B 97 X-D$ functional has been tested in the calculations of the binding energies of the benzene dimer. The binding energies for three conformations of the benzene dimer, T-shaped $(T)$, parallel displaced $(P D)$ and sandwich $(S)$ (see the structures in ref. 40, Fig. 1), calculated using several different density functionals and basis sets are presented and compared with the literature data in Table 1. In the literature, there is no consensus about the global minimum structure of the benzene dimer. The $\mathrm{MP} 2+\Delta \mathrm{CCSD}(\mathrm{T})$ results for the estimated basis set limit interaction energy, ${ }^{40}$ as well as a DFT-based description, ${ }^{41}$ show a preference for the $P D$ conformation over the $T$ structure. However, the DFT results with empirical dispersion correction generally predict that the $T$ geometry is 
(a)

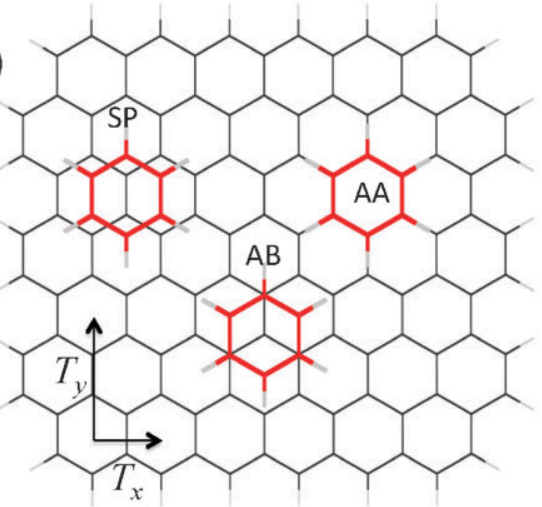

(b)

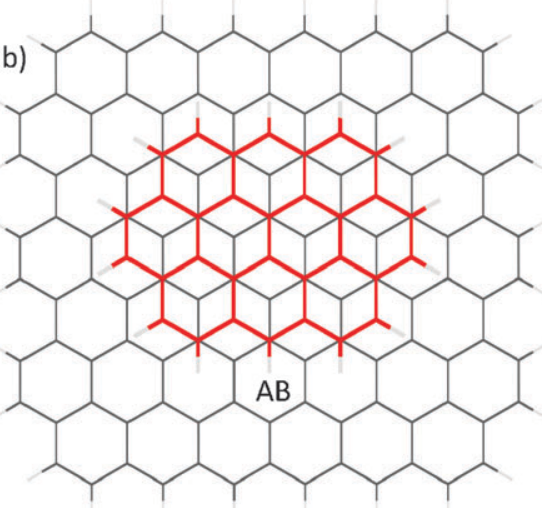

Fig. 1 High symmetry positions of PAHs on the hydrogen-terminated graphene showing: (a) the benzene molecule in the AA, AB, and SP positions; (b) the ovalene molecule in the $\mathrm{AB}$ position. The translational lengths of the graphene lattice are $T_{x}=2.46 \AA$, and $T_{y}=4.26 \AA$.

most stable. This is consistent with the results for the binding energies obtained with the B97-D and $\omega$ B97X-D functionals, as shown in Table 1. The B97-D functional differs from the $\omega \mathrm{B} 97 \mathrm{X}-\mathrm{D}$ functional in the form of the damping function used and in the absence of a short-range Hartree-Fock correction. Within the GGA (BLYP functional ${ }^{42,43}$ ) approach, all three conformations of the benzene dimer remain unbound. The LDA (SVWN functional ${ }^{44}$ ) predictions for the binding of the benzene dimer are reasonable, similar to the case of the interlayer binding in graphite described in the introduction, however the binding energies for the $S$ and $P D$ conformations are underestimated. The LC hybrid density functional $\omega \mathrm{B} 97 \mathrm{X}$ significantly underestimates the binding for all three conformations. Hence LDA, GGA and LC hybrid DFT functionals without empirical dispersion corrections should be recognized as inadequate for the description of binding in graphitic structures.

The $S$ conformation is less stable than the $P D$ structure, and the difference in the binding energies, $\Delta E^{P D-S}$, of these two parallel conformations is typically about $30-40 \mathrm{meV}$. Remarkably, $\Delta E^{P D-S}$ does not vary as much as the binding energies with computational method. Even the GGA (BLYP) approach with $\mathrm{TZ}(\mathrm{d}, \mathrm{p})$ basis set, while giving no binding whatsoever, predicts the value of $\Delta E^{P D-S}$ only two times larger than the value obtained with the $\omega$ B97X-D functional and the same basis set. $\triangle E^{P D-S}$ is essentially analogous to the energy barrier separating two minima of the interaction potential energy surface (PES) calculated as a function of the relative motion of the walls of carbon nanotubes or the layers of graphene.
However, while the intermolecular interaction in the benzene dimer tends to zero at large lateral displacement, in ideal infinite graphitic materials the relative motion of neighbouring layers is defined by the oscillatory PES with equivalent minima attributed to the symmetry of the system.

\section{Adsorption and motion of polycyclic aromatic hydrocarbons on graphene}

\section{A. Binding energy}

An atomic cluster description of the interacting system of a PAH with the hydrogen-terminated graphene sheet consisting of 116 carbon atoms $\left(\mathrm{C}_{116} \mathrm{H}_{28}\right)$ has been used. In each case, $\mathrm{PAH}$ is initially placed on the substrate in the so-called $\mathrm{AB}$ stacking which corresponds to the position at which half of the carbon atoms of PAH are located directly above the carbon atoms of the graphene sheet and the other half are facing the center of hexagons of the substrate. The AB position of $\mathrm{PAH}$ on the substrate corresponds to the global minimum in PES, and it is shown in Fig. 1 for the smallest (benzene-hydrogen terminated graphene, Fig. 1a) and the largest (ovalene-hydrogen terminated graphene, Fig. 1b) systems studied. Table 2 presents the comparison between the experimental binding energies of PAHs with the surface of graphite and the theoretical binding energies between PAHs and hydrogen-terminated graphene in the $\mathrm{AB}$ position calculated at the $\omega \mathrm{B} 97 \mathrm{X}-\mathrm{D} / \mathrm{STO}-3 \mathrm{G}$, $\omega B 97 X-D / 6-31 G^{*}, \omega B 97 X / S T O-3 G$, and $\omega$ B97X/6-31G* levels of theory. For each interacting system, the geometry in the

Table 1 The BSSE corrected binding energies (in meV) for $T$-shaped $\left(E^{T}\right)$, parallel-displaced $\left(E^{P D}\right)$ and sandwich $\left(E^{S}\right)$ conformations of the benzene dimer calculated using several computational methods. $\Delta E^{P D-S}$ is the energy barrier defined as the difference in the binding energies of the $P D$ and $S$ structures

\begin{tabular}{|c|c|c|c|c|c|}
\hline Method & Basis set & $E^{T}$ & $E^{S}$ & $E^{P D}$ & $\Delta E^{P D-S}$ \\
\hline $\mathrm{MP} 2+\Delta \operatorname{CCSD}(\mathrm{T})^{40}$ & CBS limit & 119 & 79 & 121 & 42 \\
\hline B97-D ${ }^{39}$ & TZV2P & 130 & 77 & 119 & 42 \\
\hline$\omega B 97 X-D$ & $\mathrm{TZ}(\mathrm{d}, \mathrm{p})$ & 116 & 80 & 107 & 27 \\
\hline$\omega B 97 X-D$ & $6-311++\mathrm{G}(3 \mathrm{df}, 3 \mathrm{pd})$ & 126 & 94 & 115 & 21 \\
\hline$\omega B 97 X-D$ & $6-31 G^{*}$ & 116 & 49 & 80 & 31 \\
\hline$\omega \mathrm{B} 97 \mathrm{X}$ & $\mathrm{TZ}(\mathrm{d}, \mathrm{p})$ & 86 & 24 & 55 & 31 \\
\hline LDA(SVWN) & $\mathrm{TZ}(\mathrm{d}, \mathrm{p})$ & 128 & 37 & 88 & 51 \\
\hline GGA(BLYP) & $\mathrm{TZ}(\mathrm{d}, \mathrm{p})$ & -73 & -187 & -132 & 55 \\
\hline
\end{tabular}


Table 2 The binding energies (in eV) of the benzene, napthalene, coronene and ovalene molecules on graphite surface (experiment $\left.{ }^{8}\right)$ and on the hydrogen-terminated graphene substrate $\mathrm{C}_{116} \mathrm{H}_{28}$ (theory, this work). The computed values of the binding energies (BSSE corrected) correspond to the geometries of the interacting systems in the $\mathrm{AB}$ position optimized at the $\omega \mathrm{B} 97 \mathrm{X}-\mathrm{D} / \mathrm{STO}-3 \mathrm{G}$ level of theory

\begin{tabular}{|c|c|c|c|c|c|c|}
\hline PAH & $\begin{array}{l}\text { Experiment, } \\
\text { Redhead analysis }^{45}\end{array}$ & $\begin{array}{l}\text { Experiment }^{8}, \\
\text { Falconer-Madix analysis }\end{array}$ & $\begin{array}{l}\omega \mathrm{B} 97 \mathrm{X}-\mathrm{D} / \\
6-31 \mathrm{G}^{*}\end{array}$ & $\begin{array}{l}\omega \mathrm{B} 97 \mathrm{X}-\mathrm{D} / \\
\text { STO-3G }\end{array}$ & $\begin{array}{l}\omega \mathrm{B} 97 \mathrm{X} / \\
6-31 \mathrm{G}^{*}\end{array}$ & $\begin{array}{l}\omega B 97 X / \\
\text { STO-3G }\end{array}$ \\
\hline $\mathrm{C}_{6} \mathrm{H}_{6}$ & $0.50 \pm 0.08$ & $0.50 \pm 0.08$ & 0.47 & 0.37 & 0.13 & -0.05 \\
\hline $\mathrm{C}_{10} \mathrm{H}_{8}$ & $0.8 \pm 0.1$ & $0.90 \pm 0.07$ & 0.76 & 0.59 & 0.21 & -0.07 \\
\hline $\mathrm{C}_{24} \mathrm{H}_{12}$ & $1.3 \pm 0.2$ & $1.5 \pm 0.1$ & 1.73 & 1.29 & 0.47 & -0.14 \\
\hline $\mathrm{C}_{32} \mathrm{H}_{14}$ & $2.2 \pm 0.2$ & $1.97 \pm 0.08$ & 2.23 & 1.69 & 0.61 & -0.18 \\
\hline
\end{tabular}

$\mathrm{AB}$ position has been optimized using $\omega \mathrm{B} 97 \mathrm{X}-\mathrm{D} / \mathrm{STO}-3 \mathrm{G}$ approach. The use of larger basis sets was computationally prohibitive for these large systems. The optimized values of the $z$-separation between PAHs and the $\mathrm{C}_{116} \mathrm{H}_{28}$ substrate are tabulated in Table 3, it can be seen that in the minimum $\mathrm{AB}$ position the $z$-separation grows with increase of the molecule size.

The binding energy of the adsorbate to the graphite surface can be identified with the activation energy for desorption, as measured by thermal desorption (TD) spectroscopy. ${ }^{8}$ The rate of desorption of an adsorbate from a solid surface is commonly described by the Arrhenius equation. Based on the Arrhenius equation, a number of techniques has been proposed that allow the determination of the activation energies for desorption of an adsorbate using the series of the TD spectra. These include an analysis using the Redhead's peak maximum method $^{45}$ and an isothermal analysis of Falconer and Madix. ${ }^{46}$ The first numerical column of Table 2 gives estimations of the activation energy for desorption of PAHs on the graphite surface using the Readhead analysis which relates the energy to the temperature at the desorption peak maximum $\left(T_{\max }\right)$ and the heating rate obtained in experiment. ${ }^{8}$ The second numerical column of Table 2 is the result of an isothermal analysis of the TD spectra using the Falconer-Madix method which employs a linear fit to isothermal desorption data obtained in the experiment ${ }^{8}$ in the temperature range used.

The next four columns of Table 2 contain the calculated BSSE corrected binding energies. The $\omega$ B97X-D/STO-3G results underestimate the experimental values, however in all four cases a sufficient binding is predicted. The improved values obtained at the $\omega \mathrm{B} 97 \mathrm{X}-\mathrm{D} / 6-31 \mathrm{G}^{*}$ level of theory are in good general agreement with experiment. The calculated $\omega \mathrm{B} 97 \mathrm{X}-\mathrm{D} / 6-31 \mathrm{G}^{*}$ values for the binding energies of coronene and ovalene molecules are somewhat greater than the experimental predictions. There could be a number of possible reasons for the discrepancy. The TD spectra of benzene and napthalene exhibit two clearly distinguishable desorption peaks corresponding to monolayer and multilayer desorption allowing unambiguous determination of $T_{\max }$. On the other

Table 3 The values of $z$-separation (in $\AA$ ) between PAHs and the hydrogen-terminated graphene substrate $\mathrm{C}_{116} \mathrm{H}_{28}$ in the $\mathrm{AA}, \mathrm{AB}$ and SP stacking obtained at the $\omega$ B $97 \mathrm{X}-\mathrm{D} / \mathrm{STO}-3 \mathrm{G}$ level of theory

\begin{tabular}{llll}
\hline PAH & AB & AA & SP \\
\hline $\mathrm{C}_{6} \mathrm{H}_{6}$ & 3.35 & 3.36 & 3.30 \\
$\mathrm{C}_{10} \mathrm{H}_{8}$ & 3.36 & 3.37 & 3.34 \\
$\mathrm{C}_{24} \mathrm{H}_{12}$ & 3.38 & 3.40 & 3.31 \\
$\mathrm{C}_{32} \mathrm{H}_{14}$ & 3.40 & 3.41 & 3.33 \\
\hline
\end{tabular}

hand, desorption traces of coronene and ovalene exhibit a behaviour indicative of fractional order kinetics, and desorption features of the first monolayer in the TD spectra cannot be clearly distinguished from the multilayer peaks. This leaves considerable uncertainty in the determination of the monolayer desorption maxima and hence desorption temperatures. Additionally, as the data on the density of ovalene (the number of adsorbate molecules per unit area) adsorbed on the graphite surface is not available from low energy electron diffraction or scanning tunneling microscopy experiments, an estimated value was used in ref. 8 . It should be also noted that the calculated values correspond to the binding of PAHs to a graphene layer, not bulk graphite.

The binding predicted by the $\omega \mathrm{B} 97 \mathrm{X}$ functional is poor, indicating that the long-range van der Waals interactions, the dominant contribution to the binding between PAHs and the graphene substrate, cannot be described sufficiently well using this functional. Note that the $\omega \mathrm{B} 97 \mathrm{X} / \mathrm{STO}-3 \mathrm{G}$ calculations consistently give unbound results. In all considered cases, the values for the binding energy calculated with $\omega \mathrm{B} 97 \mathrm{X}-\mathrm{D} /$ 6-31G* are approximately 3.65 times larger than the values predicted by $\omega \mathrm{B} 97 \mathrm{X} / 6-31 \mathrm{G}^{*}$.

\section{B. Interaction energy profiles}

In addition to global minimum defining the binding energies, PES for the interaction of a PAH with graphene as a function of the molecular displacement along the substrate has two other types of critical points which correspond to the high symmetry orientations of the molecular ring on the substrate. Global maximum corresponds to a position at which all of the atoms in neighbouring layers face each other, so-called AA stacking. Saddle points are associated with the relative orientation lying between the equivalent positions corresponding to the closest minima, which has a similarity with the $P D$ conformation of the benzene dimer, and is called SP stacking. Fig. 1a shows the high symmetry positions of the benzene molecule on graphene which correspond to the critical points of PES. The energy difference in the AA and AB positions defines the largest barrier in the energy profile for the relative sliding of a PAH along the substrate, whereas the energy difference between the AB and SP positions gives a secondary corrugation in PES.

Table 4 presents the results for the energy barriers to motion of PAHs along the graphene substrate, calculated at the centre of a large $\mathrm{C}_{116} \mathrm{H}_{28}$ flake in order to minimize edge effects. To allow a direct comparison of the energy barriers obtained for different molecules, the values are given in $\mathrm{meV}$ per carbon atom of PAH. Similarly to the calculations of the binding 
Table 4 The energy barriers (in meV per carbon atom of PAH) between the global minimum and the global maximum (AB-AA), and the global minimum and the saddle point (AB-SP) of the effective interaction potential energy surface. The geometries of the interacting systems in the AA, $\mathrm{AB}$ and SP positions have been optimized at the $\omega \mathrm{B} 97 \mathrm{X}-\mathrm{D} / \mathrm{STO}-3 \mathrm{G}$ level of theory

\begin{tabular}{|c|c|c|c|c|c|c|c|c|}
\hline \multirow[b]{2}{*}{ PAH } & \multicolumn{2}{|c|}{$\omega \mathrm{B} 97 \mathrm{X}-\mathrm{D} / 6-31 \mathrm{G}^{*}$} & \multicolumn{2}{|c|}{$\omega \mathrm{B} 97 \mathrm{X}-\mathrm{D} / \mathrm{STO}-3 \mathrm{G}$} & \multicolumn{2}{|c|}{$\omega \mathrm{B} 97 \mathrm{X} / 6-31 \mathrm{G}^{*}$} & \multicolumn{2}{|c|}{$\omega \mathrm{B} 97 \mathrm{X} / \mathrm{STO}-3 \mathrm{G}$} \\
\hline & AB-AA & AB-SP & AB-AA & AB-SP & AB-AA & AB-SP & AB-AA & AB-SP \\
\hline $\mathrm{C}_{6} \mathrm{H}_{6}$ & 9.4 & 2.2 & 5.1 & 3.2 & 9.4 & 5.0 & 4.7 & 4.8 \\
\hline $\mathrm{C}_{10} \mathrm{H}_{8}$ & 9.5 & 2.4 & 5.3 & 2.1 & 9.3 & 4.1 & 4.8 & 3.3 \\
\hline $\mathrm{C}_{24} \mathrm{H}_{12}$ & 11.0 & 3.1 & 5.1 & 3.3 & 10.6 & 6.7 & 5.0 & 5.6 \\
\hline $\mathrm{C}_{32} \mathrm{H}_{14}$ & 10.9 & 2.8 & 5.5 & 3.0 & 10.4 & 6.5 & 4.9 & 5.2 \\
\hline
\end{tabular}

energies, for each interacting system geometry optimisation was initially performed using the $\omega$ B97X-D/STO-3G level of theory (see Table 3 for the optimized values of $z$-separation), and the optimised geometries were subsequently used to calculate the barriers to motion of a molecule along the substrate using $\omega$ B97X and $\omega$ B97X-D functionals with STO-3G and $6-31 G^{*}$ basis sets. The values obtained for the interaction energies are BSSE corrected for each relative position of the interacting system.

The values for the largest energy barrier of the AB-AA transition are close for all considered PAHs, regardless of the functional used. Hence, the $\omega$ B97X-D energy barrier in the infinite graphene bilayer can be estimated as having the value of approximately $\Delta V_{\text {int }}=11 \mathrm{meV} /$ atom. Comparison of the values for the barriers calculated with ( $\omega$ B97X-D) and without $(\omega \mathrm{B} 97 \mathrm{X})$ taking into account the dispersion interactions shows that inclusion of the dispersion term does not affect the results obtained for the main barrier. The ratio for the AB-AA barriers obtained with the $\omega \mathrm{B} 97 \mathrm{X}-\mathrm{D}$ and $\omega \mathrm{B} 97 \mathrm{X}$ functionals using the $6-31 \mathrm{G}^{*}$ basis set is 1.00 for $\mathrm{C}_{6} \mathrm{H}_{6}, 1.02$ for $\mathrm{C}_{10} \mathrm{H}_{8}$, 1.03 for $\mathrm{C}_{24} \mathrm{H}_{12}$, and 1.04 for $\mathrm{C}_{32} \mathrm{H}_{14}$. It can therefore be concluded that the inclusion of the dispersion interaction correction as an additional empirical term to the DFT treatment does not change the values of the major barrier to the relative motion of PAHs on graphene.

If the graphene substrate is assumed to be a rigid infinite lattice with a multitude of 6-fold axes of symmetry, the interaction between a single carbon atom in $\mathrm{PAH}$ and graphene can be approximated using the following analytical potential ${ }^{47}$

$$
V_{\text {int }}^{C}(x, y, z)=V_{1}(z)-V_{0}(z)\left[2 \cos \left(a_{x} x\right) \cos \left(a_{y} y\right)+\cos \left(2 a_{y} y\right)\right] \text {, }
$$

which represents the lowest harmonics of the Fourier expansion of PES. ${ }^{48}$ The analytical potential (5) is defined such that $x=0$ and $y=0$ correspond to the position at which a single carbon atom is located directly above the centre of hexagon in graphene; $V_{0}(z)$ is the height-dependent surface corrugation amplitude, and $V_{1}(z)$ determines the position-averaged $z$-dependence of the interaction. The parameters $a_{x}$ and $a_{y}$ are defined by the periodicity of the graphene lattice as $a_{x}=2 \pi / T_{x}$ and $a_{y}=2 \pi / T_{y}=a_{x} / \sqrt{3}$, where $T_{x}$ and $T_{y}$ are the translational lengths of the lattice in the perpendicular $x$ - and $y$-directions shown in Fig. 1. If the carbon-carbon bond is taken to be $1.42 \AA$ then $T_{x}=2.46 \AA$, and $T_{y}=4.26 \AA$. If $\mathrm{PAH}$ is considered to be a rigid molecule, the interaction potential between the substrate and $\mathrm{PAH}$ can be obtained by summation of all contributions from the PAH atoms. The effective potential for the movement of the centre of mass of PAH (CM) on graphene, calculated per carbon atom of $\mathrm{PAH}$, has the following form

$$
\begin{aligned}
V_{\text {int }}^{\mathrm{PAH}}\left(x_{t}, y_{t}, z_{t}\right)= & V_{1}\left(z_{t}\right)-V_{0}\left(z_{t}\right)\left[\cos \left(a_{x} x_{t}\right) \cos \left(a_{y} y_{t}+\frac{\pi}{3}\right)\right. \\
& \left.-\frac{1}{2} \cos \left(2 a_{y} y_{t}+\frac{2 \pi}{3}\right)\right] .
\end{aligned}
$$

The effective potential (6) is determined such that the position of CM for which $x_{t}=0$ and $y_{t}=0$ corresponds to the AB stacking as shown in Fig. 1. The contributions of hydrogen atoms to the interaction potential have been neglected.

To allow a comparison with the analytical profile (6) for the interaction potential energy as a function of the molecular displacement along the substrate, a series of calculations have been produced of the BSSE-corrected $\omega \mathrm{B} 97 \mathrm{X}-\mathrm{D} / 6-31 \mathrm{G}^{*}$ interaction energies between the rigid $\mathrm{C}_{6} \mathrm{H}_{6}$ and $\mathrm{C}_{116} \mathrm{H}_{28}$ molecules separated by $z=3.35 \AA$, the value close to the experimental separation in graphite ${ }^{49}$ and to the distances obtained in the geometry optimisation calculations (see Table 3). The carboncarbon bond length was fixed at $1.42 \AA$. The results of such comparison are presented in Fig. 2. The displacement of benzene along the substrate is first calculated by placing the molecule in the AB stacking position in the middle of the fixed graphene flake, and then translating it along the $y$-direction through the AB-AA-AB-SP-AB path. The interaction potential profile which corresponds to the continuous displacement along the AB-AA-AB-SP-AB path is denoted by triangles in Fig. 2, and the analytical profile obtained with the use of eqn (6) is represented by a solid line.

\section{Dispersion contribution to the interaction energy}

Fig. 2 shows that for continuous displacement of the benzene molecule along the AB-AA-AB-SP-AB path of the $\mathrm{C}_{116} \mathrm{H}_{28}$ flake the small secondary energy barrier corresponding to the AB-SP transition is overestimated compared to the case of motion of the benzene molecule along an infinite graphene layer. The discrepancy can be explained by the edge effects in the interaction with a finite substrate that affect the dispersion contribution to the interaction energy. In order to examine the behaviour of the dispersion energy $E_{\text {disp }}$ as a function of displacement on a surface, the empirical component of the $\omega \mathrm{B} 97 \mathrm{X}-\mathrm{D}$ dispersion energy was calculated using eqn (3) for several series of interactions. The behaviour of the empirical component of the $\omega \mathrm{B} 97 \mathrm{X}-\mathrm{D}$ dispersion energy is qualitatively similar to the one used by Grimme in the DFT-D schemes, ${ }^{39}$ which only differ by the use of an overall scaling factor and the $\mathrm{Wu}-\mathrm{Yang}^{50}$ damping function. Thus, the investigation of the 


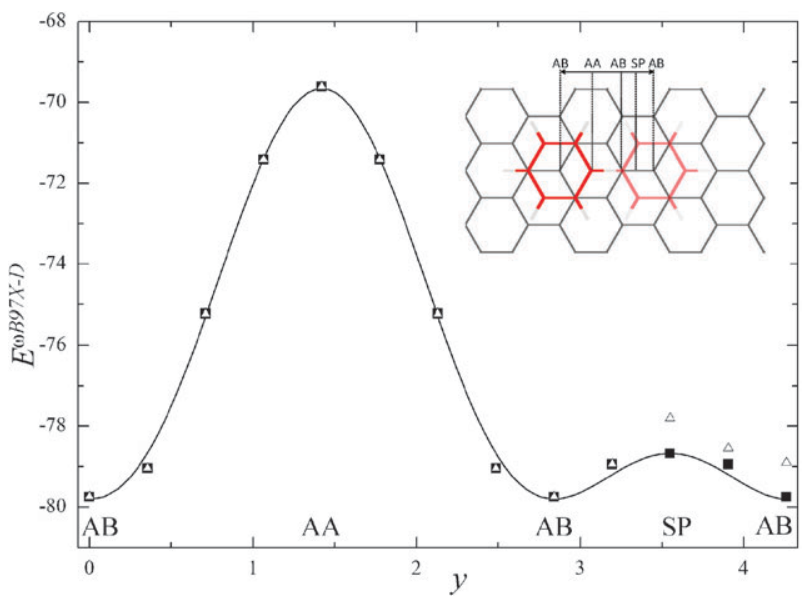

Fig. 2 The interaction potential energy of the benzene- $\mathrm{C}_{116} \mathrm{H}_{28}$ system as a function of the benzene displacement along the AB-AAAB-SP-AB path (shown in the inset) calculated using $\omega B$ 97X-D/ 6-31G* level of theory, as described in the text. Energy (BSSE corrected) is given in $\mathrm{meV}$ per carbon atom of the benzene molecule; distance $y$ is given in $\AA$; $z$ separation is fixed at $3.35 \AA$. The profile obtained using eqn (6) is shown by solid line; the calculated values of the interaction energy along the AB-AA-AB-SP-AB path are depicted by empty triangles showing the edge effects, mainly in the estimation of the empirical dispersion term; the values of the interaction energy calculated at the centre of the $\mathrm{C}_{116} \mathrm{H}_{28}$ flake are shown in filled squares.

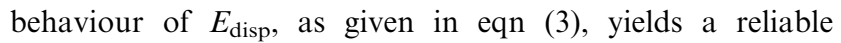
estimation of the dispersion correction to the DFT energy.

The series of dispersion energy profiles containing the benzene molecule interacting with larger molecules is shown in Fig. 3. The displacement, $D$, is calculated by initially fixing the $\mathrm{CM}$ of benzene directly over the $\mathrm{CM}$ of the larger molecules $(D=0)$. For the benzene-coronene system and the benzene- $\mathrm{C}_{116} \mathrm{H}_{28}$ substrate system, $D=0$ corresponds to the AA stacking position at which the $\mathrm{CM}$ of both molecules is located in the centre of a ring. For the benzene-naphthalene and benzene-ovalene systems, $D=0$ corresponds to the SP stacking position at which the CM of benzene is in the centre of a ring and the other molecule is bond-centred. The profiles are then calculated by translating the benzene molecule along the $y$ axis by $20 \AA$ in both directions. The profile showing the dispersion energy of the benzene dimer is also included in Fig. 3 for completeness. For the interaction of benzene with the largest molecule considered, $\mathrm{C}_{116} \mathrm{H}_{28}$, the dispersion energy profile has an approximately $10 \AA$ wide plateau centred at $D=0$, which indicates that the dispersion contribution to the interaction energy does not change significantly in the middle of the large flake compared to the edge (the dimensions of the $\mathrm{C}_{116} \mathrm{H}_{28}$ substrate are 17.2 and $15.6 \AA$ in the $x$ - and $y$ - directions, correspondingly, without taking hydrogens into consideration). A similar flat profile of the dispersion energy in the vicinity of $D=0$ was obtained for larger PAHs, as shown in Fig. 4, with the plateau being narrowest for the ovalene$\mathrm{C}_{116} \mathrm{H}_{28}$ system. The dispersion energy profiles for the interaction between two identical PAHs, however, show no such feature (Fig. 5).

Even small variations in $E_{\text {disp }}$ affect the value of the secondary energy barrier shown in Fig. 2. From the inset of

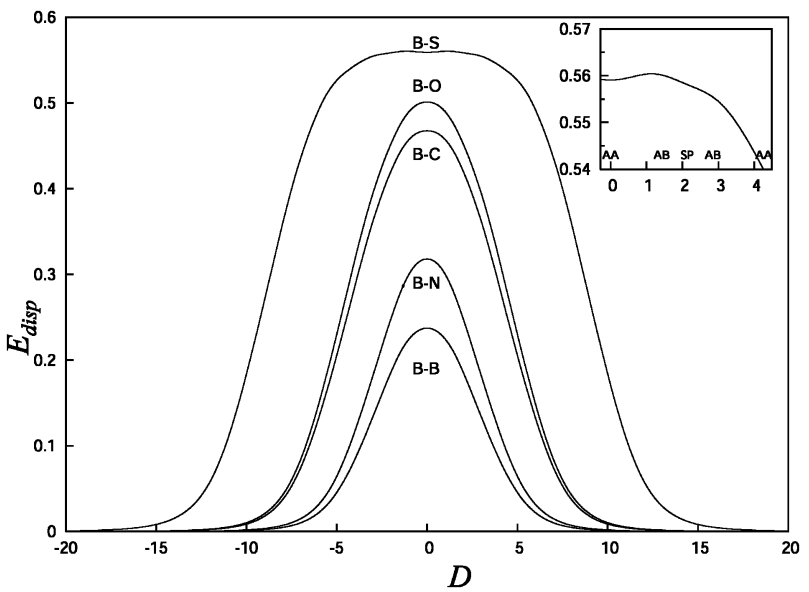

Fig. 3 The dispersion energy $E_{\text {disp }}$ (in eV) calculated using formula (3) as a function of the relative displacement of molecules, $D$ (in $\AA$ ), as described in the text. $\mathrm{B}-\mathrm{S}$ : benzene- $\mathrm{C}_{116} \mathrm{H}_{28}$ substrate; $\mathrm{B}-\mathrm{O}$ : benzene-ovalene; $\mathrm{B}-\mathrm{C}$ : benzene-coronene; $\mathrm{B}-\mathrm{N}$ : benzene-naphthalene; and B-B: benzene dimer. The inset shows the first AA-AB-SP-AB-AA transition for the B-S system.

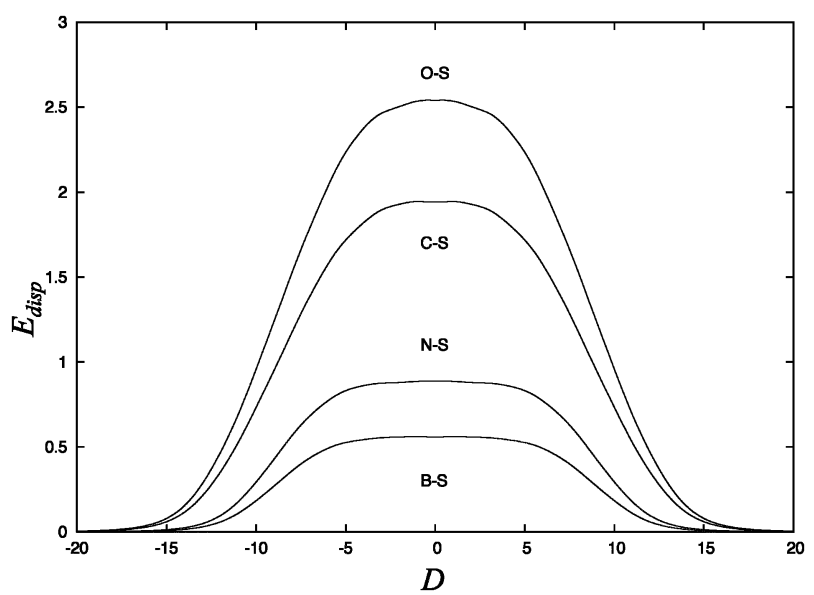

Fig. 4 The dispersion energy $E_{\text {disp }}$ (in eV) calculated using formula (3) as a function of the relative displacement of molecules, $D$ (in $\AA$ ), as described in the text. O-S: ovalene- $\mathrm{C}_{116} \mathrm{H}_{28} ; \mathrm{C}-\mathrm{S}$ : coronene- $\mathrm{C}_{116} \mathrm{H}_{28}$; $\mathrm{N}-\mathrm{S}$ : naphthalene- $\mathrm{C}_{116} \mathrm{H}_{28}$; and $\mathrm{B}-\mathrm{S}$ : benzene- $\mathrm{C}_{116} \mathrm{H}_{28}$ molecule.

Fig. 3, it can be estimated that the difference in the dispersion energy values obtained in the $\mathrm{AB}$ positions along the AB-SP-AB path is $\Delta E_{\text {disp }}=4.7 \mathrm{meV}$. The energy difference in the total interaction potential energy profile calculated in the equivalent $\mathrm{AB}$ positions along the AB-SP-AB path (triangles in Fig. 2) is $\Delta E^{\omega \mathrm{B} 97 \mathrm{X}-\mathrm{D}}=5.1 \mathrm{meV}$. This indicates that the difference of $5.1 \mathrm{meV}$ in the values of the interaction potential energy in the equivalent $\mathrm{AB}$ positions is a result of the edge effects in estimation of the empirical dispersion term. The edge effects can be eliminated if the interaction energy is calculated not along the continuous path from the centre of the flake towards the edge but in the positions at the centre of the flake which correspond to the same relative orientations of PAH and substrate as those along the AB-SP-AB path. This approach (the profile denoted by squares in Fig. 2) gives the values of the large AB-AA barrier and the small AB-SP barrier of $10.1 \mathrm{meV}$ and $1.1 \mathrm{meV}$, respectively. The ratio of the 


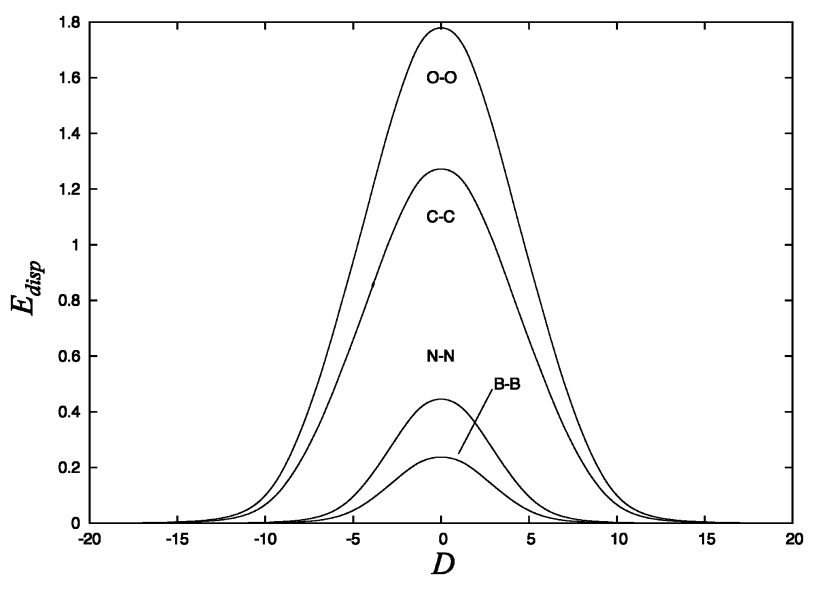

Fig. 5 The dispersion energy $E_{\text {disp }}$ (in eV) calculated using formula (3) as a function of the relative displacement of molecules, $D$ (in $\AA$ ), as described in the text. O-O: ovalene dimer; $\mathrm{C}-\mathrm{C}$ : coronene dimer; $\mathrm{N}-\mathrm{N}$ : naphthalene dimer; and $\mathrm{B}-\mathrm{B}$ : benzene dimer.

barriers is about 9.2 which is very close to the analytical value of 9 .

The potential amplitude $V_{0}$ in eqn (5) describing the interaction of a single carbon atom with graphene was found ${ }^{47}$ to be in the region between 3.3 and $6.7 \mathrm{meV}$ by fitting to the experimental results on the atomic-scale friction forces between a small flake and graphene. ${ }^{51}$ In the profile for the interaction potential energy of the benzene- $\mathrm{C}_{116} \mathrm{H}_{28}$ system calculated using formula (6) and presented in Fig. 2 by solid line, the value of $V_{0}$ is $4.5 \pm 0.1 \mathrm{meV} /$ atom, which falls well within the estimated experimental range. It follows from eqn (6) that the barrier between the global minimum and global maximum of the potential energy profile is $2.25 V_{0}$. Hence, the experimental range for the values of the AB-AA energy barrier for the interaction of PAHs with graphene can be estimated as 7.5-15 meV, and the data presented in Table 4 are in excellent agreement with the experimental range.

\section{Friction forces}

The calculated PES for the interaction of PAHs on graphene can also be directly used in estimation of the friction forces acting on the molecules along the direction of motion. The frictional mechanisms between small graphene flakes and graphite have been studied experimentally using a frictional force microscope that achieves a resolution in the lateral forces down to $15 \mathrm{pN}^{51}$ There was found to be a strong dependence of friction on orientation of the molecules, in which two narrow peaks of high friction were observed at flake-substrate orientation (rotation) angles of 0 and $60^{\circ}$. The first peak had a maximum friction force of $306 \pm 40 \mathrm{pN}$, and the second peak had a maximum of $203 \pm 20 \mathrm{pN}$. Between these peaks, a wide angular range of ultra-low friction close to the detection limit of the microscope was found, thus providing evidence of the superlubricity of graphite. The friction force is maximal when the orientation angle, which defines the mismatch between the lattices of a flake and substrate, is $\Phi=0^{\circ}$ or $60^{\circ}$ (in accordance with $60^{\circ}$ rotational symmetry of a graphite sheet), i.e. the flake slides over the graphite surface in commensurate contact ${ }^{47,51}$. In this geometry, the flake as a whole
Table 5 The frictional forces between PAHs and the hydrogenterminated graphene (in $\mathrm{pN}$ ) calculated using formula (7) along the $\mathrm{AB}-\mathrm{AA}-\mathrm{AB}$ and $\mathrm{AB}-\mathrm{SP}-\mathrm{AB}$ pathways

\begin{tabular}{lll}
\hline & AB-AA-AB & AB-SP-AB \\
\hline $\mathrm{C}_{6} \mathrm{H}_{6}$ & 64 & 30 \\
$\mathrm{C}_{10} \mathrm{H}_{8}$ & 107 & 54 \\
$\mathrm{C}_{24} \mathrm{H}_{12}$ & 298 & 168 \\
$\mathrm{C}_{32} \mathrm{H}_{14}$ & 394 & 202 \\
\hline
\end{tabular}

performs a slip-stick motion due to the binding of a flake to the graphite in the $\mathrm{AB}$ stacking relative configuration. The ultra-low friction behaviour and enhanced slipperiness occurs when the flake slides over the graphite surface in incommensurate contact. The flake is then rotated out of perfect registry causing the kinetic friction force to vanish and preventing the collective stick-slip motion of all atoms in the contact. The experimentally observed variation of the lateral friction force with the periodicity of graphene lattice fits very well the theoretical data provided by a modified Tomlinson model. ${ }^{51}$

The effective PES calculated in this paper correspond to the commensurate case of matching lattices $\left(\Phi=0^{\circ}\right)$. If the flake is then moved along the AB-AA-AB and AB-SP-AB pathways on the graphene surface such that the stacking of layers was maintained at $\Phi=0^{\circ}$, the frictional force $F_{f}$ can be simply evaluated as ${ }^{52}$

$$
F_{f}=\frac{\Delta V_{\mathrm{int}} N_{c}}{a},
$$

where $\Delta V_{\text {int }}$ is the value of the energy barriers to the sliding of PAHs on graphene taken from Table $4, N_{c}$ is the number of carbon atoms in contact with substrate, and the parameter $a$ is the spacing between the minimum (AB stacking) and maximum (AA or SP stacking) on the PES, having the value of $1.42 \AA$ for the motion along the AB-AA-AB pathway and $0.71 \AA$ along the AB-SP-AB pathway. The calculated values of the frictional forces for four considered PAHs are presented in Table 5. According to Verhoeven et al., ${ }^{47}$ the flake during the interaction with graphene only visits limited areas of the PES, namely those flat regions of the surface in the immediate proximity to the minima of the PES. The AB-SP-AB path is energetically more favourable, and hence the friction forces along it (second numerical column of Table 5) correspond to the slip-stick motion during which energy is dissipated thus causing friction, while the highest fiction force can only be achieved by pulling the flake through the AB-AA-AB path with a microscope tip. It can be seen from Table 5 that the friction force increases almost linearly with the number of atoms in the contact.

\section{Summary}

This study of the interaction of PAHs with a single layer of hydrogen-terminated graphene has been stimulated by recent TEM experiments on fragmentation of graphene sheets into small graphitic flakes, ${ }^{33}$ whose subsequent interaction with graphene substrate has not yet been investigated in details. The DFT-D method has been chosen as it provides a satisfactory balance between the accuracy of predictions and 
computational cost. The selected $\omega \mathrm{B} 97 \mathrm{X}-\mathrm{D}$ functional was previously tested on a variety of weakly bound systems, ${ }^{36}$ and produced results comparable to the common DFT-D functionals B97-D, B3LYP-D, and BLYP-D. ${ }^{39}$ In this paper, $\omega \mathrm{B} 97 \mathrm{X}-\mathrm{D}$ was used to estimate the binding energies of three conformations of the benzene dimer, and the results obtained with the large $\mathrm{TZ}(\mathrm{d}, \mathrm{p})$ and $6-311++\mathrm{G}(3 \mathrm{df}, 3 \mathrm{pd})$ basis sets showed a good agreement with the literature data.

The potential energy surfaces for the interaction of benzene, $\mathrm{C}_{6} \mathrm{H}_{6}$, naphthalene, $\mathrm{C}_{10} \mathrm{H}_{8}$, coronene, $\mathrm{C}_{24} \mathrm{H}_{12}$, and ovalene, $\mathrm{C}_{32} \mathrm{H}_{14}$, with graphene were calculated as functions of the PAH displacement along the hydrogen-terminated graphene substrate. The computed binding energies are in agreement with the experimental data measured by thermal desorption spectroscopy ${ }^{8}$ and analysed with both Redhead's peak maximum method $^{45}$ and an isothermal analysis of Falconer and Madix. ${ }^{46}$ The movement of PAHs on graphene was also described analytically using the lowest harmonics of the Fourier expansion of PES, both computational and analytical results are in excellent agreement. Comparison of the values for the barriers to the movement of PAHs on graphene calculated with and without taking into account the dispersion interactions shows that inclusion of the dispersion term does not change the shape of the interaction energy surfaces or the value of the barriers. For all four considered PAHs, the ratio for the main AB-AA barrier obtained with the $\omega$ B97X-D and $\omega$ B97X functionals remains consistently close to unity. The potential energy surfaces were further used in the estimation of the friction forces acting on the molecules along the direction of motion, which compares favourably with experiments studying the frictional mechanisms between graphitic flakes and graphite using a frictional force microscopy. ${ }^{51}$

\section{Acknowledgements}

E.B. gratefully acknowledges financial support of an EPSRC Career Acceleration Fellowship. This work was performed during O.E.'s visit to the University of Nottingham funded by Scholarship of the President of Russian Federation for education abroad. O.E. acknowledges financial support of Russian Foundation for basic research (grant 08-02-00685-a), and thanks her supervisors Dr A. M. Popov and Prof. Y. E. Lozovik for useful discussions.

\section{References}

1 S. Grimme, J. Comput. Chem., 2004, 25, 1463.

2 S. Tsuzuki, K. Honda, T. Uchimura and M. Mikami, J. Chem. Phys., 2004, 120, 647.

3 S. Tsuzuki and H. P. J. Luethi, J. Chem. Phys., 2001, 114, 3949.

4 U. Zimmerli, M. Parrinello and P. J. Koumoutsakos, J. Chem. Phys., 2004, 120, 2693.

5 M. Allen and D. J. Tozer, J. Chem. Phys., 2002, 117, 11113.

6 L. X. Benedict, N. G. Chopra, M. L. Cohen, A. Zettl, S. G. Louie and V. H. Crespi, Chem. Phys. Lett., 1998, 286, 490.

7 L. A. Girifalco and R. A. Lad, J. Chem. Phys., 1956, 25, 693.

8 R. Zacharia, H. Ulbricht and T. Hertel, Phys. Rev. B: Condens. Matter Mater. Phys., 2004, 69, 155406.

9 M. Hasegawa, K. Nishidate and H. Iyetomi, Phys. Rev. B: Condens. Matter Mater. Phys., 2007, 76, 115424.
10 T. Gould, K. Simpkins and J. F. Dobson, Phys. Rev. B: Condens. Matter Mater. Phys., 2008, 77, 165134.

11 M. Hasegawa and K. Nishidate, Phys. Rev. B: Condens. Matter Mater. Phys., 2004, 70, 205431.

12 H. Rydberg, N. Jacobson, P. Hyldgaard, S. I. Simak, B. I. Lundqvist and D. C. Langreth, Surf. Sci., 2003, 606, 532.

13 J. C. Charlier, X. Gonze and J. P. Michenaud, Europhys. Lett., 1994, 28, 403.

14 M. C. Schabel and J. L. Martins, Phys. Rev. B: Condens. Matter, 1992, 46, 7185.

15 S. B. Trickey, F. Muller-Plathe, G. H. F. Diercksen and J. C. Boettger, Phys. Rev. B: Condens. Matter, 1992, 45, 4460.

16 R. H. Telling and M. I. Heggie, Philos. Mag. Lett., 2003, 83, 411.

17 H. Rydberg, M. Dion, N. Jacobson, E. Schroder, P. Hyldgaard, S. I. Simak, D. C. Langreth and B. Lundqvist, Phys. Rev. Lett., 2003, 91, 126402.

18 L. A. Girifalco and M. Hodak, Phys. Rev. B: Condens. Matter Mater. Phys., 2002, 65, 125404.

19 F. Ortmann, F. Bechstedt and W. G. Schmidt, Phys. Rev. B. Condens. Matter Mater. Phys., 2006, 73, 205101.

20 V. V. Deshpande, H.-Y. Chiu, H. W. Ch. Postma, C. Miko, L. Forro and M. Bockrath, Nano Lett., 2006, 6, 1092.

21 B. Bourlon, D. C. Glattli, C. Miko, L. Forro and A. Bachtold, Nano Lett., 2004, 4, 709.

22 J. Cumings and A. Zettl, Science, 2000, 289, 602.

23 A. Kis, K. Jensen, S. Aloni, W. Mickelson and A. Zettl, Phys. Rev. Lett., 2006, 97, 025501.

24 L. Forro, Science, 2000, 289, 560.

25 A. M. Fennimore, T. D. Yuzvinsky, W.-Q. Han, M. S. Fuhrer, J. Cumings and A. Zettl, Nature, 2003, 424, 408.

26 A. Barreiro, R. Rurali, E. R. Hernandez, J. Moser, T. Pichler, L. Forro and A. Bachtold, Science, 2008, 320, 775.

27 Q. Zheng, B. Jiang, S. Liu, Y. Weng, L. Lu, Q. Xue, J. Zhu, Q. Jiang, S. Wang and L. Peng, Phys. Rev. Lett., 2008, 100, 067205 .

28 E. Bichoutskaia, A. M. Popov, Y. E. Lozovik, O. V. Ershova, I. V. Lebedeva and A. A. Knizhnik, Phys. Rev. B: Condens. Matter Mater. Phys., 2009, 80, 165427.

29 E. Bichoutskaia, Philos. Trans. R. Soc. London, Ser. A, 2007, 365, 2893.

30 E. Bichoutskaia, A. M. Popov, A. El-Barbary, M. I. Heggie and Y. E. Lozovik, Phys. Rev. B: Condens. Matter Mater. Phys., 2005, 71, 113403.

31 E. Bichoutskaia, A. M. Popov, M. I. Heggie and Y. E. Lozovik, Phys. Rev. B: Condens. Matter Mater. Phys., 2006, 73, 045435.

32 A. K. Geim and K. S. Novoselov, Nat. Mater., 2007, 6, 183.

33 A. Chuvilin, U. Kaiser, E. Bichoutskaia, N. A. Besley and A. N. Khlobystov, Nature Chemistry, 2010, in press.

34 J. Kong, C. A. White, A. I. Krylov, C. D. Sherrill, R. D. Adamson, T. R. Furlani, M. S. Lee, A. M. Lee, S. R. Gwaltney, T. R. Adams, C. Ochsenfeld, A. T. B. Gilbert, G. S. Kedziora, V. A. Rassolov, D. R. Maurice, N. Nair, Y. Shao, N. A. Besley, P. E. Maslen, J. P. Dombroski, H. Daschel, W. Zhang, P. P. Korambath, J. Baker, E. F. C. Byrd, T. Van Voorhis, M. Oumi, S. Hirata, C.-P. Hsu, N. Ishikawa, J. Florian, A. Warshel, B. G. Johnson, P. M. W. Gill, M. Head-Gordon and J. A. Pople, J. Comput. Chem., 2000, 21, 1532.

35 S. F. Boys and F. Bernardi, Mol. Phys., 1970, 19, 553.

36 J.-D. Chai and M. Head-Gordon, Phys. Chem. Chem. Phys., 2008, 10, 6615 .

37 J.-D. Chai and M. Head-Gordon, J. Chem. Phys., 2008, 128, 084106.

38 A. D. Becke, J. Chem. Phys., 1997, 107, 8554.

39 S. Grimme, J. Comput. Chem., 2006, $27,1787$.

40 M. O. Sinnokrot and C. D. Sherrill, J. Phys. Chem. A, 2004, 108, 10200.

41 T. Sato, T. Tsuneda and K. Hirao, J. Chem. Phys., 2005, 123, 104307.

42 A. D. Becke, Phys. Rev. A: At., Mol., Opt. Phys., 1988, 38, 3098.

43 C. Lee, W. Yang and R. G. Parr, Phys. Rev. B: Condens. Matter, 1988, 37, 785.

44 S. J. Vosko, L. Wilk and M. Nusair, Can. J. Phys., 1980, 58, 1200.

45 P. A. Redhead, Vacuum, 1962, 12, 203.

46 J. L. Falconer and R. J. Madix, J. Catal., 1977, 48, 262. 
47 G. S. Verhoeven, M. Dienwiebel and J. W. M. Frenken, Phys. Rev. B: Condens. Matter Mater. Phys., 2004, 70, 165418.

48 W. A. Steele, Surf. Sci., 1973, 36, 317.

49 Y. Baskin and L. Meyer, Phys. Rev., 1955, 100, 544.

50 Q. Wu and W. Yang, J. Chem. Phys., 2002, 116, 515.
51 M. Dienwiebel, G. S. Verhoeven, N. Pradeep, J. W. M. Frenken, J. A. Heinberg and H. W. Zandbergen, Phys. Rev. Lett., 2004, 92, 126101.

52 K. Miura, N. Sasaki and S. Kamiya, Phys. Rev. B: Condens. Matter Mater. Phys., 2004, 69, 075420. 